\title{
Prophage-encoded chitinase activity supports growth of a Pseudomonas sp isolated from Kermadec Trench
}

\author{
SACHIA J TRAVING ${ }^{1}$, DANIEL CASTILLO ${ }^{2}$, RONNIE N \\ GLUD $^{1}$ AND MATHIAS MIDDELBOE ${ }^{3}$ \\ ${ }^{1}$ University of Southern Denmark \\ ${ }^{2}$ Universidad SEK \\ ${ }^{3}$ University of Copenhagen \\ Presenting Author: sjtraving@biology.sdu.dk
}

The number of microbes we currently have in culture make up just a small fraction of their diversity in natural ecosystems, yet culturable representatives provide invaluable knowledge needed for understanding microbial and viral ecology and as reference material for validating gene functions for sequence data. This is also true for hadal systems where microbial and viral sequence data reveal high sequence novelty with little homology to known gene functions.

Here we present the first characterizations of a phage-Pseudomonas sp. system isolated from the Kermadec Trench at $\sim 9.300 \mathrm{~m}$ depth. The virus genome is $\sim 40 \mathrm{kbp}$ in size with 52 open reading frames and encodes an integrase and a chitinase gene. The virus is therefore temperate and $>95 \%$ of host cells isolated from sediment samples were lysogenic and contained the phage genome as a prophage. The host and phage carry two different chitinase genes, suggesting that chitin degradation is key for their survival in the trench. Comparisons between lysogenic and non-lysogenic host strains demonstrated that the prophage, stimulated growth and chitinase activity of the Pseudomonas host in the presence of chitin. Further, the prophage induction rate correlated negatively with chitin concentration, suggesting that induction rate was regulated by substrate conditions. Overall, the results indicate that the prophage-encoded chitinase supports chitin degradation and growth by the lysogenic host, and that the host can regulate prophage induction when chitin is present. The work illustrates the importance of prophage-encoded genes for host performance and emphasizes the potential role of virus-host interactions for bacterial cycling of organic matter in deep sea environments. 